\title{
Length-area-volume of long bubbles in horizontal slug flow
}

\author{
L.M.C. Matamoros ${ }^{a}$, J.B.R. Loureiro ${ }^{\mathrm{a}, \mathrm{b}, *}$, A.P. Silva Freire ${ }^{\mathrm{a}}$ \\ ${ }^{a}$ Mechanical Engineering Program (PEM/COPPE/UFRJ), C.P. 68503, 21941-972 Rio de Janeiro, Brazil \\ ${ }^{\mathrm{b}}$ Mechanical Engineering Department (DEM/Poli/UFRJ), C.P. 68503, 21941-972 Rio de Janeiro, Brazil
}

\section{A R T I C L E I N F O}

\section{Article history:}

Received 3 January 2014

Received in revised form 17 March 2014

Accepted 13 May 2014

Available online 22 May 2014

\section{Keywords:}

Horizontal slug flow

Interface

Fractal dimension

\begin{abstract}
A B S T R A C T
The morphology of long bubbles in horizontal slug flows is experimentally investigated through images obtained with a high-speed camera. The flow conditions resulted in very long and interface-perturbed bubbles, so that a special procedure needed to be developed to correctly identify all of their geometric features. The procedure furnishes a complete characterization of the three-dimensional aspects of the nose, main body, hydraulic jump and tail of bubbles, including their fractal dimension and gas-liquid interface area. Image treatment and numerical computations were carried out with Wolfram's Mathematica 8.0 software system. The results are compared with a previously advanced theory. Characteristic volumes and areas of long bubbles in slug flows are correlated through simple power-law expressions.
\end{abstract}

(c) 2014 Elsevier Ltd. All rights reserved.

\section{Introduction}

The description of multiphase flows depends essentially on the correct representation of surfaces and interfaces. Yet, the complexity of most practical problems normally forces analyses to consider interfaces as smooth surfaces, deprived of wrinkles, folds and roughness. While this may be a good approximation for small bubbles, drops or particles, departures from this condition may incur in large discrepancies, with a particular impact on the estimation of interface areas.

Recent oil discoveries in Brazil have disclosed the existence of large reservoirs in ultradeep sea waters, with unprecedented large concentrations of $\mathrm{CO}_{2}$ and $\mathrm{H}_{2} \mathrm{~S}$. In this scenario, the prevalent flow pattern in the production lines is expected to be slug flow, with the dominant effect of very long bubbles. The influx of a soluble gas into the wellbore is expected to cause profound changes on the properties of slug flows, which, therefore, must be correctly addressed.

Magalhaes et al. (2013) have discussed the changes in $\mathrm{CO}_{2}$ water slug flow properties due to solubility effects. Shadow Sizer (SS) and Particle Image Velocimetry (PIV) were used to determine the size distributions of the liquid slug and of the long bubbles, the liquid velocity in the slug, the velocity in the liquid film, the

\footnotetext{
* Corresponding author at: Mechanical Engineering Program (PEM/COPPE/UFRJ), C.P. 68503 , 21941-972 Rio de Janeiro, Brazil. Tel.: +55 2136223548; fax: +55 2136223552.

E-mail address: jbrloureiro@gmail.com (J.B.R. Loureiro).
}

velocity of propagation of the nose of the long bubbles and the pressure drop. Two expressions that correlate length with interface area and volume were advanced and incorporated into onedimensional mechanistic models for horizontal slug flows. These quantities were assessed from simple geometric arguments, based on two reference parameters: the total length of the bubble and the average chord of the cross section of the liquid film.

The present technical note characterizes the length, interface area and volume of long bubbles in slug flow through a detailed description of their typical four regions: nose, main body, hydraulic jump and tail. The discrepancy between experiments and the theory of Fagundes Netto et al. (1999) for the prediction of the interface area of certain regions (nose and tail) are found to be as high as $40 \%$. For very long bubbles the average error on the theoretical estimation of the interface area is about 6\%. The work also introduces the fractal dimension of the projected contour of bubbles on the axial measurement plane. For nose and tail, the average fractal dimensions $\left(D_{K}\right)$ were found to be 1.24 and 1.25 , respectively. For the main body, average $D_{K}$ is 1.16 .

Of course, the classical mechanistic models that have been developed for the description of slug flow do not consider the fine details of the shape of bubbles. To determine the two-dimensional contour of bubbles, the most comprehensive theoretical study in literature is possibly that of Fagundes Netto et al. (1999) which has not been independently validated. This is briefly done here.

The chief experimental technique of the present work is the Shadow Sizer technique. Images acquired at high frequencies were processed to obtain the desired geometric properties of bubbles. The high resolution of the images unveils the complexities of the 
interface between the gas and liquid phases, showing how local instabilities produce sharp fluctuations on the bubble interface.

To incorporate interface effects into one-dimensional theories, the simplest possibility is to develop expressions of the form $l=f\left(V^{1 / 3}\right)$ and $A=g\left(V^{2 / 3}\right)$, where $l$ stands for length, $A$ for area and $V$ for volume. In the analysis of Magalhaes et al. (2013), the total interface area and the bubble length were written as $A_{i T}=3.7 V^{2 / 3}$ and $L_{f}=271 V^{2 / 3}-2.4 V^{1 / 3}$, respectively. The present very detailed study suggests otherwise that: $A_{i T} / D^{2}=$ $3.70\left(V^{2 / 3} / D^{2}\right)^{1.16}$ and $L_{f} / D=3.12\left(V^{1 / 3} / D\right)^{2.48}$, where $D$ denotes the pipe diameter.

\section{The distorted surface of large bubbles}

Bubbles with volumes greater than about $3 \mathrm{~cm}^{3}$ are frequently considered large bubbles. Fig. 1 shows the effects of interface instabilities on two different bubbles: a spherical-cap bubble and a horizontal slug flow bubble.

In case (a), the continuous phase is at rest. Instabilities sweep down the interface and tend to grow through the Rayleigh-Taylor instability mechanism (Clift et al., 2005). Surface tension and viscous effects tend to damp the growth of instabilities on the unstable surface, hampering breakup. The maximum stable size of air bubbles in water in an unbounded environment is about $5 \mathrm{~cm}$.

In case (b), the bubble is exposed to large velocity gradients and turbulence. Under this condition, the local shear stress and the energy of eddies of size comparable to the bubble size are the primary source of instabilities.

Bubbles of the latter type are those that are considered in the present work. Their shapes are known to depend on the mixture mean velocity and also be independent of the bubble length. In fact, the motion of a slug flow bubble is governed almost entirely by the dynamics of the nose and tail (Wallis, 1969). Henceforth, the experimental results are presented in terms of Froude number based on the mixture mean velocity.

The contours of the noses of two horizontal slug flow bubbles are shown in Fig. 2. The shapes are represented in terms of the liquid volume fraction $\left(\alpha_{L}\right)$ as a function of the normalized distance to the leading edge of the bubble nose $(\xi=x / D)$. The perturbations provoked by the oncoming flow $(\varphi=V-U)$ result in a distorted surface with fractal dimension of about 1.24 . Here, $V$ stands for

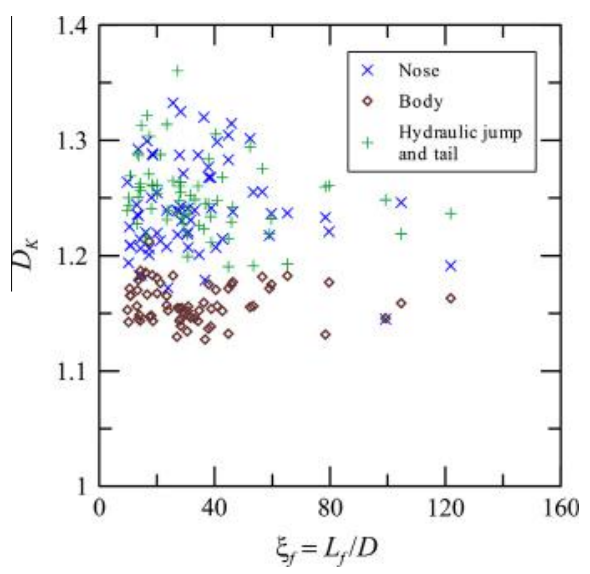

Fig. 3. Fractal dimensions of the nose, body and tail of horizontal slug flow bubbles.

the bubble velocity and $U\left(=\left(Q_{l}+Q_{g}\right) / A_{c}\right)$ for the mixture mean velocity, where $Q_{l}$ and $Q_{g}$ are the liquid and gas volumetric flow rates, respectively, and $A_{c}$ is the cross-sectional area of the pipe.

The fractal dimensions of the nose, body and tail of bubbles are shown in Fig. 3. These data were compiled through the box counting method (Duboc et al., 1989) and the experimental data set is described below in the following sections.

\section{Experiment}

Experimental analysis is possibly the only feasible manner to understand the fundamentals of complex turbulent two-phase flows and develop mechanistic predictive models. Typical flow parameters including phase velocities, pressure, characteristic frequencies and temperatures, need to be incorporated into theories, and the information obtained from experiments has played a leading role on establishing important empirical relations that, coupled with the first principles, produce workable models.

Many important flow variables, including velocity and typical frequency, were measured in the past through intrusive techniques. The work of Fagundes Netto et al. (1999), for example, used

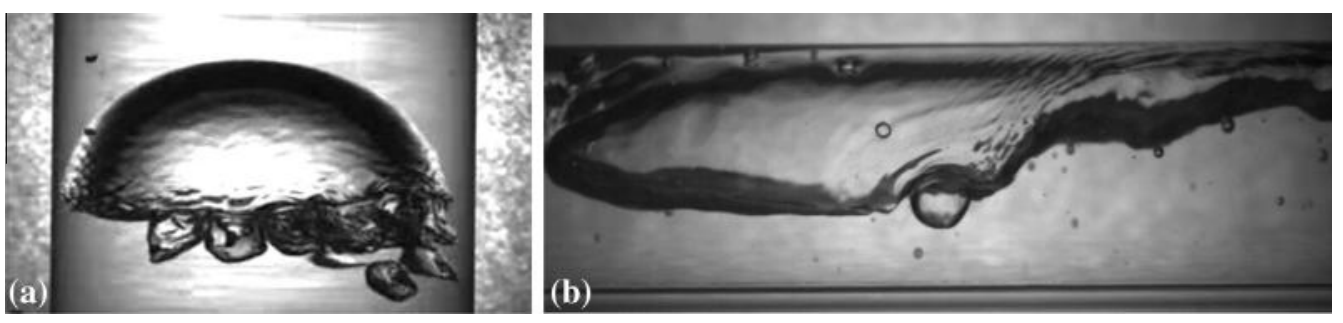

Fig. 1. Surface instabilities: (a) spherical-cap bubble, (b) short horizontal slug flow bubble.
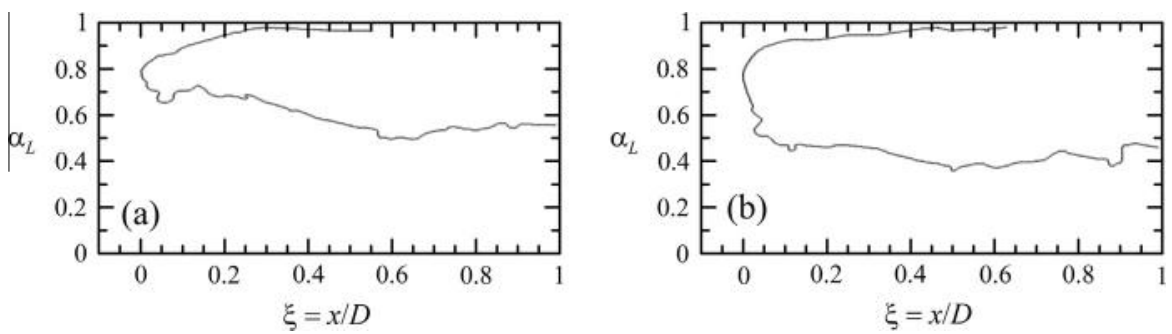

Fig. 2. Nose contour: (a) $V=2.0 \mathrm{~m} \mathrm{~s}^{-1}, U=1.67 \mathrm{~m} \mathrm{~s}^{-1}$, and (b) $V=2.29 \mathrm{~m} \mathrm{~s}^{-1}, U=1.90 \mathrm{~m} \mathrm{~s}^{-1}$. 
conductance and capacitance probes. These are invasive techniques that alter the properties of a flow. An alternative technique, as shown by Mayor et al. (2007), is the use of high speed cameras.

With the recent advances in digital electronics, new measuring devices have been developed, which specially ensure the access to data with great spatial and temporal resolution. One of these devices is the high-speed digital camera. Depending on the model, more than two thousand images per second can be acquired. This feature has recently become very important to applications in scientific and technological development, so much as one considers the recent advances in image treatment. The analysis of multiphase flows greatly benefited in the past from classical high-speed filming, but the recent advances in image treatment have allowed new characteristics to be explored.

Mayor et al. (2007) used digital images to calculate velocities, lengths of bubbles and liquid slugs in vertical pipes. In this work, different processes for the treatment of images were discussed, including gray scale conversion, filtering, subtraction, conversion to binary, erosion and the definition of the bubble shape. The work also developed expressions for the estimation of the uncertainties of some flow parameters.

The work of Mayor et al. (2007) has basically determined the linear dimensions of bubbles. The present work estimates areas and volumes from two-dimensional measurements.

\section{Test facility}

The experiments were carried out in the Laboratory of Multiphase Flows in Pipes (LEMT) of the Mechanical Engineering Program of the Federal University of Rio de Janeiro. The basic set up consists of a horizontal acrylic pipe of $45.7 \mathrm{~mm}$ in diameter and $12 \mathrm{~m}$ in length. Water is stored in a $4 \mathrm{~m}^{3}$ tank and a positive displacement pump of $20 \mathrm{~m}^{3} \mathrm{~h}^{-1}$ maximum flow rate is used to feed the fluid to the test section. The gas phase is provided by an air compressor of $100 \mathrm{~m}^{3} \mathrm{~h}^{-1}$ maximum flow rate. A Coriolis water flow meter and an air rotameter were used to monitor and control the test conditions. The Dantec Shadow Sizer system is composed by a NanoSense Mk III high-speed camera with acquisition rate of 2080 frames per second and resolution of $1280 \times 1024$ pixels. A Constellation LED light source was synchronized with the camera to provide still images of the bubble.

Flow patterns for $50 \mathrm{~mm}$-diameter pipes were used to define different combinations of water and air velocities that resulted in the desired slug flow configuration. The water flow velocity was kept constant and different mixture velocities were obtained by varying the air flow. As a consequence, different velocities, sizes and shapes of the bubble and liquid piston were achieved.

The experimental flow conditions are shown in Table 1 . The corresponding flow pattern map is shown Fig. 4 according to the classification of Mandhane et al. (1974).

To acquire the images, an acrylic box $(0.7 \times 0.15 \times 0.15 \mathrm{~m}$, $5 \mathrm{~mm}$ thick), was placed 8 meters downstream of the entrance. The box was filled with water to reduce image distortion.

Table 1

Experimental flow conditions.

\begin{tabular}{lll}
\hline$V\left(\mathrm{~m} \mathrm{~s}^{-1}\right)$ & $Q_{g}\left(\mathrm{~m}^{3} \mathrm{~h}^{-1}\right)$ & $\mathrm{Q}_{l}\left(\mathrm{~m}^{3} \mathrm{~h}^{-1}\right)$ \\
\hline 1.28 & 0.38 & 4 \\
1.50 & 1.38 & 4 \\
1.62 & 2.10 & 4 \\
1.84 & 2.40 & 4.9 \\
2.00 & 3.50 & 4 \\
2.29 & 5.20 & 4 \\
\hline
\end{tabular}

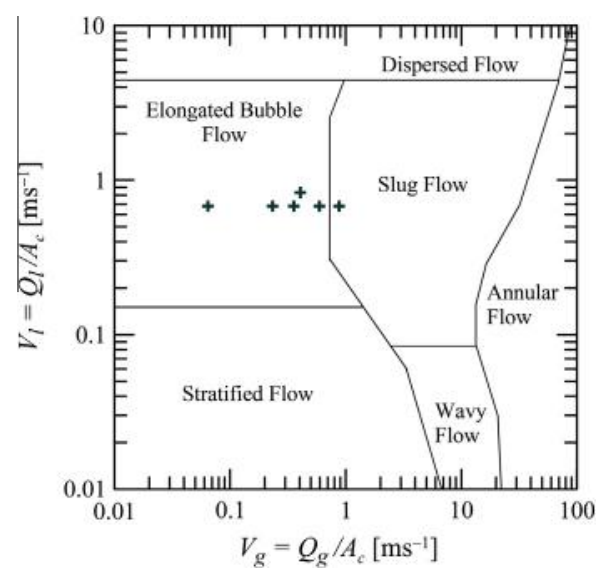

Fig. 4. Flow pattern map for the present conditions according to Mandhane et al (1974).

\section{Image treatment}

Images were acquired through the NanoSense Mk III camera at rates that varied from 2000 to 100 frames per second. Image pixels have characteristic information that can be used to define the contour of the bubbles. Given the contour of the bubbles, it is possible to define a mathematical transformation to determine the exact spatial location of each interface point and then evaluate volumes, areas and lengths. To perform the image processing, the computational platform Mathematica ${ }^{T M}$ was used.

\subsection{Evaluation of the velocity of the bubble and the frequency of image acquisition}

To evaluate the velocity of bubbles, frames were acquired at a given frequency, $v_{F}$. Images were then used to determine successive positions $\left(s_{i}\right)$ of the nose at different time steps $\left(t_{i}\right)$. Velocity was estimated from a least-square fit. When the flow is fully developed, the velocities of the nose and of the tail are almost the same.

To reconstruct the complete bubble shape, different procedures can be used (see Fig. 5). In the tracking method, the integer $N_{F}=$ IntegerPart $\left[L_{p i x} v_{F} / V_{p i x}\right]$ is used to jump across frames, selecting those that are to be matched together. The fractional part of $L_{\text {pix }} v_{F} / V_{\text {pix }}$ defines the region that must be cropped to make the adjustment right. In the present work, $L_{p i x}=1280, v_{F}$ (=frequency of acquisition) varied between 2000 and 100 , and $V_{\text {pix }}=$ bubble velocity in pixels per second. This method is the most economical in terms of the number of frames that are necessary for total bubble reconstruction.

In the fixed window approach, a reference window of width $W_{\text {pix }}$ (=IntegerPart $\left[V_{p i x} v_{F}^{-1}\right]$ ) is used to select the regions of successive frames that must be cropped and patched together. Here, the window was flush left to avoid reconstruction of the nose. This method uses all acquired frames for bubble reconstruction.

Both described procedures are not equivalent since (i) the bubbles are reconstructed from a different set of images, (ii) the tracking procedure always misses the high frequency phenomena and (iii) the fixed window procedure becomes sensitive to pixel truncation for high $v_{F}$. One obvious required condition for both methods is that the nose of the bubble remains visible over at least two consecutive frames. In the present conditions, with the lowest $v_{F}$ $(=100 \mathrm{~Hz})$, the nose position was normally observable through four consecutive frames.

Of course, if for some reason perturbations with a given high frequency $v_{p}$ must be captured, then $v_{F}$ must be at least $2 v_{p}$ (Nyquist criterion). Large frequencies are only resolved in the fixed 

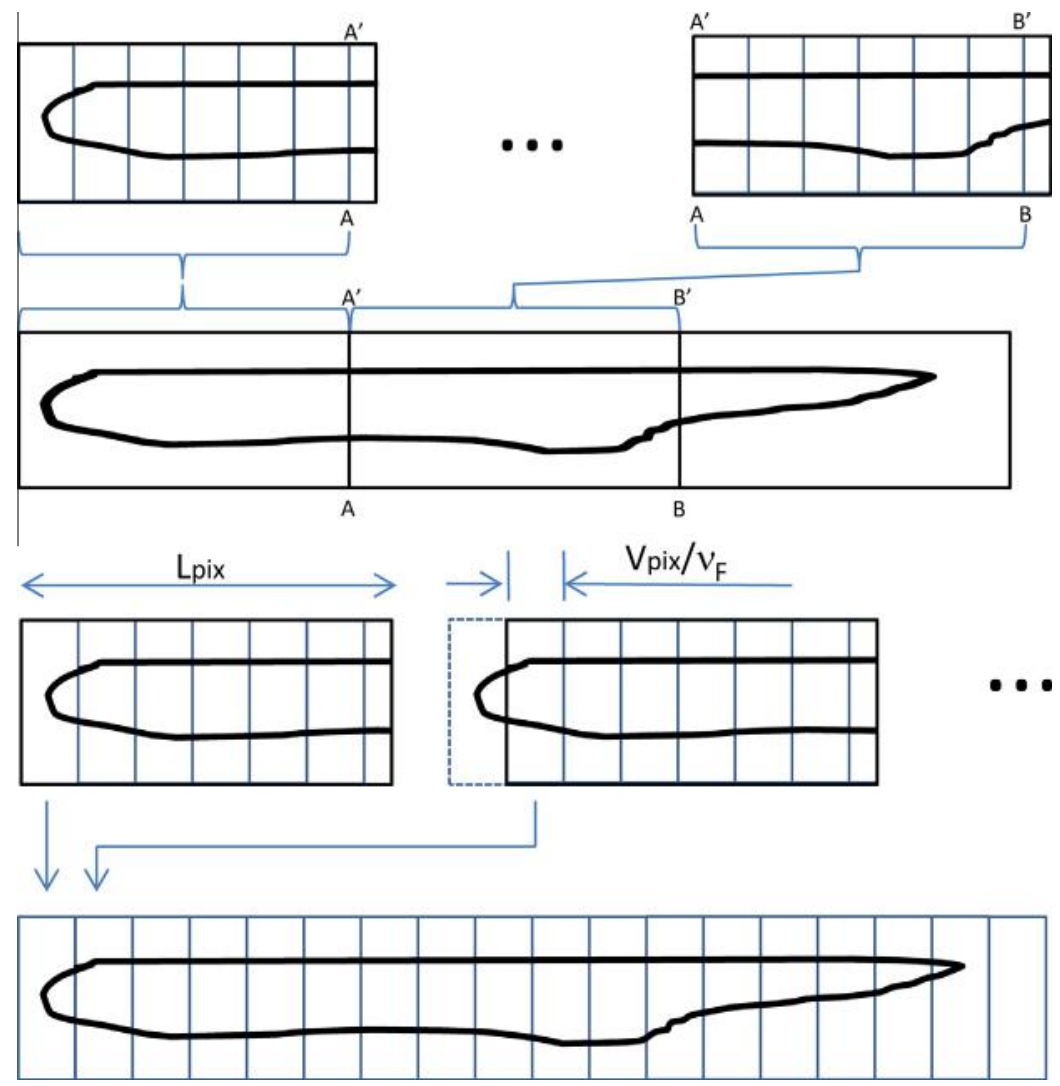

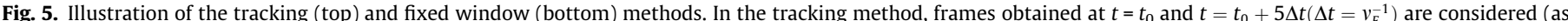

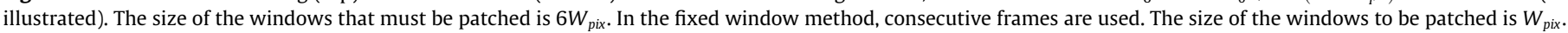
$L_{p i x}=$ frame width in pixels; $V_{p i x}=$ bubble velocity in pixels per second; $v_{F}=$ image acquisition frequency.

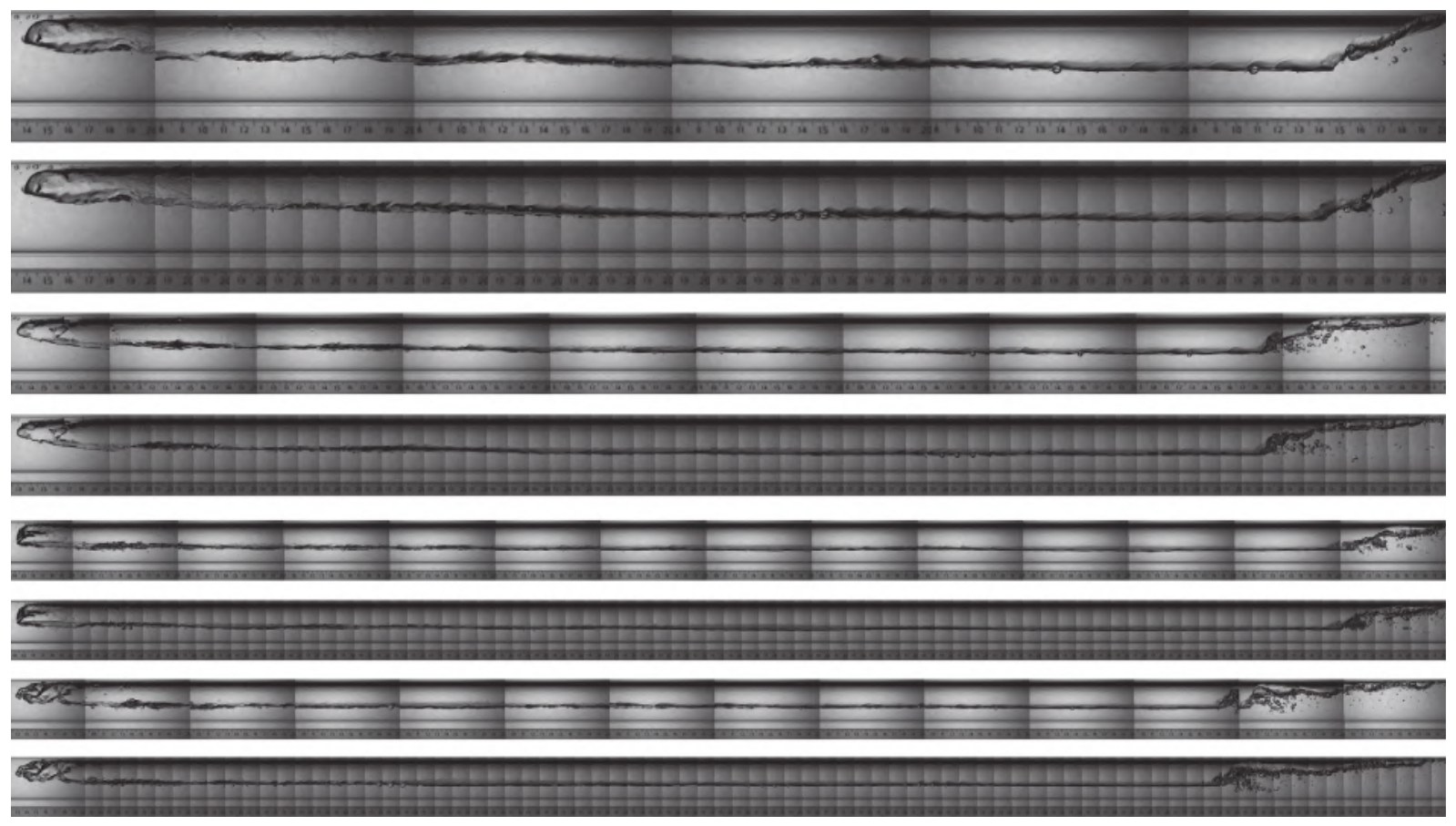

Fig. 6. Long bubbles in horizontal slug flow according to the tracking and fixed window methods $\left(v_{F}=100\right)$.

window method, which for this reason may be very demanding on data storage capacity. For example, a long bubble $\left(L_{f} / D=40\right)$ filmed at the highest frequency (in our case, $2000 \mathrm{~Hz}$ ) uses about $4 \mathrm{~Gb}$ in compacted jpeg format.
Choosing a suitable $v_{F}$ is always a critical step for bubble reconstruction. Since some of the measured bubbles were as long as $130 D$, as many as 430 images needed to be acquired to reproduce the entire bubble through the less expensive procedure. In the 
most expensive procedure this number jumped to 8700 (13 Gb of data storage). For the present experimental conditions, control of $v_{F}$ resulted sometimes in a reduction in storage space of the order of 90:1.

Typical bubble reconstruction examples for four bubbles of lengths spanning from 6 to 14 image frames (tracking method) are shown in Fig. 6. The relatively large time elapsed between frames in the tracking method induces a mismatch between image contours in consecutive frames, particularly in the tail region. On the other hand, since the image frame area is almost completely used, large spatial structures are better observed.

The steps for image processing use as basic reference the work of Mayor et al. (2007).

\subsection{Computation of areas and volume}

A full description of the hydrodynamic instability effects on horizontal slug bubbles is not within the scope of the present work. The complex interface perturbations seen in the noses and tails illustrated in Fig. 6 are difficult to characterize, both experimentally and theoretically. However, a reasonable approximation can
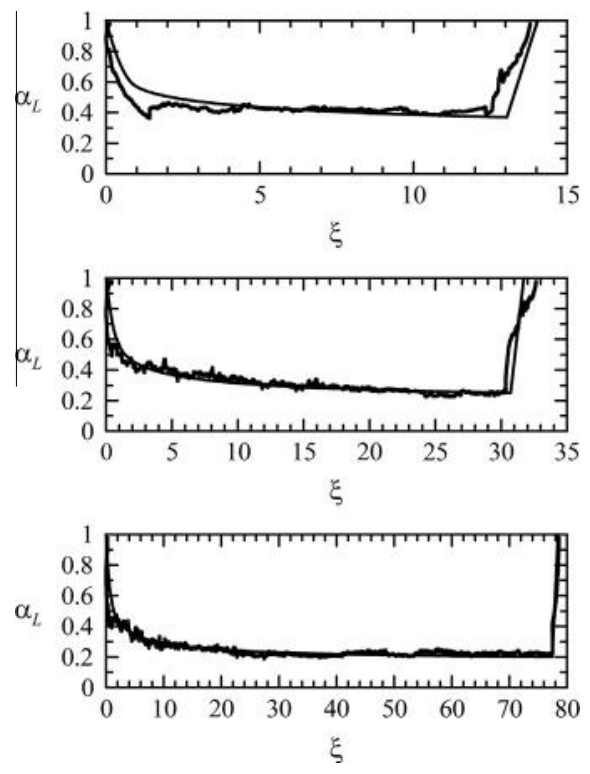

Fig. 7. The shape of a bubble for horizontal slug flow for different bubble velocities, $\xi=$ non-dimensional streamwise coordinate $(=x / D), \alpha_{L}=$ liquid volume fraction. be introduced by considering the gas-liquid interfaces to be twodimensionally distorted surfaces. Since the working pressures are low and the difference in density between water and air is very large, gravitational effects tend to keep the interface at a nearly constant level across the transversal direction. This hypothesis has also been considered by Fagundes Netto et al. (1999) to evaluate the bubble volume. Thus, perturbations are considered to propagate solely in the longitudinal direction.

With the location of the pixels, cross section slices can be defined. Once these slices are assembled together, they define the three dimensional shape of the bubble. To evaluate the areas and volume of the individual slices, simple geometric relations are used. Of course, all slices added together furnish the total area and volume of the bubble.

\section{Length-area-volume properties of long bubbles in horizontal slug flows}

Fig. 7 illustrates the type of two-dimensional contour of long bubbles that is presented in literature. The distorted line corresponds to the experimental data, whereas the smooth, well behaved line corresponds to the predictions of Fagundes Netto et al. (1999). Despite the highly distorted interface area of the bubbles, the general shapes of all three bubbles are usually well predicted. The global length is particularly well reproduced for very long bubbles with $F r_{U}<1,\left(F r_{U}=U / \sqrt{g D}\right)$.

Dramatic changes on the shape of the nose of a bubble as a function of the liquid velocity were observed. For small velocities $\left(F r_{U}<1\right)$, bubbles present a short nose located at the top of the pipe and followed by perturbations on the bottom interface characterized by instabilities with a constant wavelength and decreasing amplitude. As $\mathrm{Fr}_{U}$ increases, the nose is pushed to the center position of the pipe, increases in size and shows strong perturbations on the top and bottom interfaces.

Results for the nose interface area are shown in Fig. 8. Differences between experiments and theory are very pronounced, in average of the order of $40 \%\left(\left(A_{i n_{\text {exp }}}-A_{i n_{t h}}\right) / A_{i n_{\text {exp }}}\right)$. Fig. $8 \mathrm{~b}$ does not identify any definitive dependence of $A_{\text {in }} / D^{2}$ on $F r_{U}$. However, it is of interest to note that, despite the quantitative disagreement, qualitatively both data sets in Fig. $8 \mathrm{~b}$ tend to a maximum at about $F r_{U}=2.3$. According to Fagundes Netto et al. (1999), for $F r_{U}>2$, the wavy disturbances die out, what would explain the decrease of $A_{\text {in. }}$.

The tail structure also depends on $F r_{U}$. For low $F r_{U}$, a mild hydraulic jump followed by a well-defined tail is observed. For the higher $F r_{U}$, a tail can no longer be noted, characterizing plug flow. Instead, the hydraulic jump reaches the top of the pipe

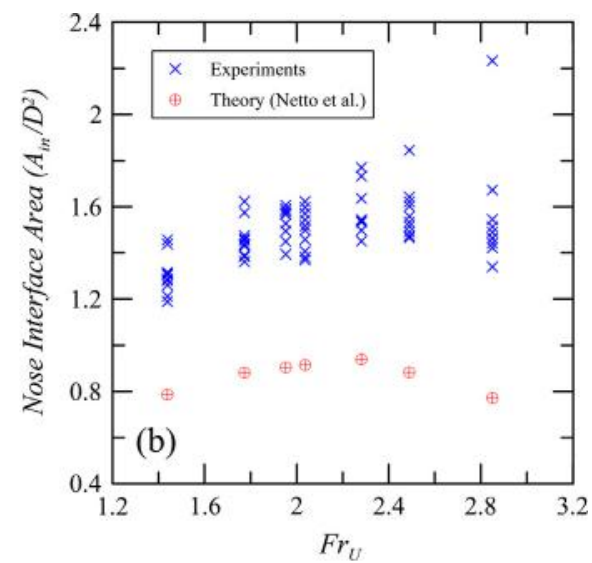

Fig. 8. Nose interface area for horizontal slug flow as a function of: (a) non-dimensional bubble length $\xi_{f}$, and (b) Froude number $F r_{U}(=U / \sqrt{g D})$. 
directly giving rise to the formation of a swarm of small bubbles. A comparison between experiments and theory for the interface area of the hydraulic jump and tail is presented in Fig. 9. The discrepancies are in average of the order of $60 \%$. Fig. 9b suggests that as $\mathrm{Fr}_{U}$ increases the difference between measurements and predictions diminishes. This result somehow agrees with the capacitance wire measurements of Fagundes Netto et al. (1999) that indicate to plug flow a less intricate bubble end interface. The disappearance of the tail for $F r_{U}>2$ reduces the available interface area at the rear of the bubble, approaching measurements and theoretical predictions.

So that interface effects can be incorporated into onedimensional theories, relations of the form $l=f\left(V^{1 / 3}\right)$ and $A=g\left(V^{2 / 3}\right)$ need to be defined, where $l$ stands for length, $A$ for the interface area and $V$ for volume.

Fig. 10 illustrates the relations between length-volume and interface area-volume for horizontal slug flows. Predictions furnished by the theory of Fagundes Netto et al. (1999) are also
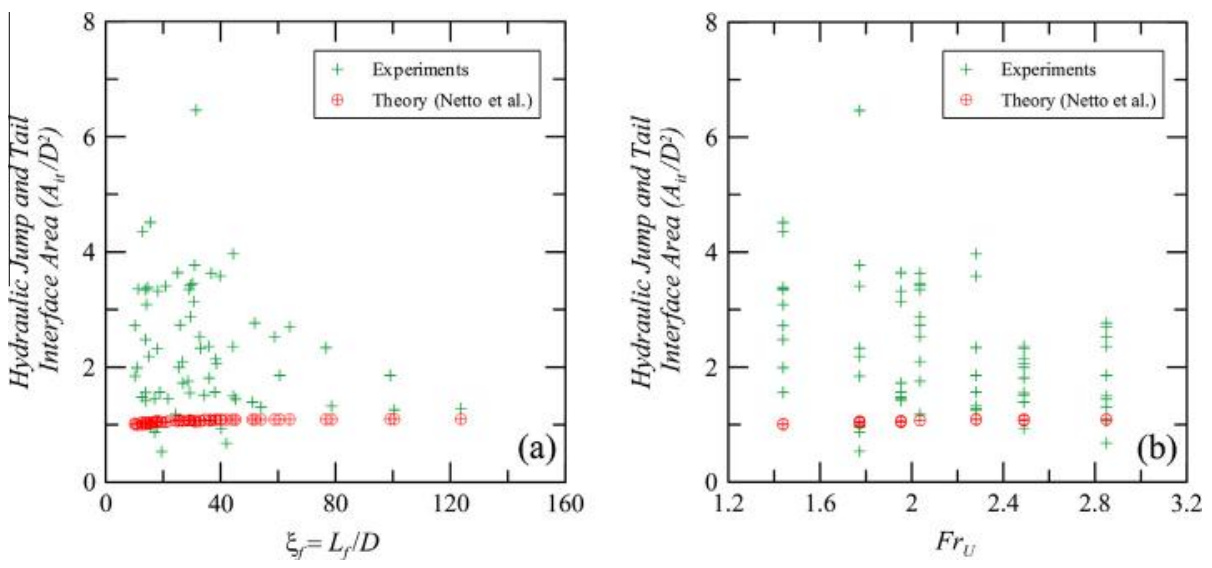

Fig. 9. Hydraulic jump and tail interface area for horizontal slug flow as a function of: (a) non-dimensional bubble length $\xi_{f}$, and $(\mathrm{b})$ Froude number $F r_{U}(=U / \sqrt{g D})$.
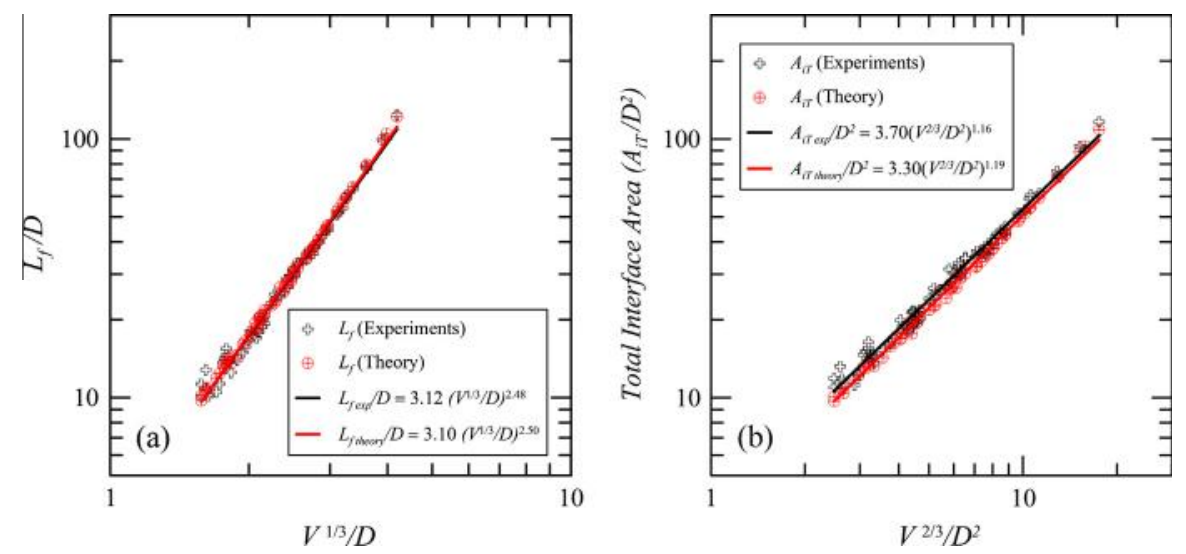

Fig. 10. Relations for horizontal slug flow: (a) length-volume and (b) interface area-volume.
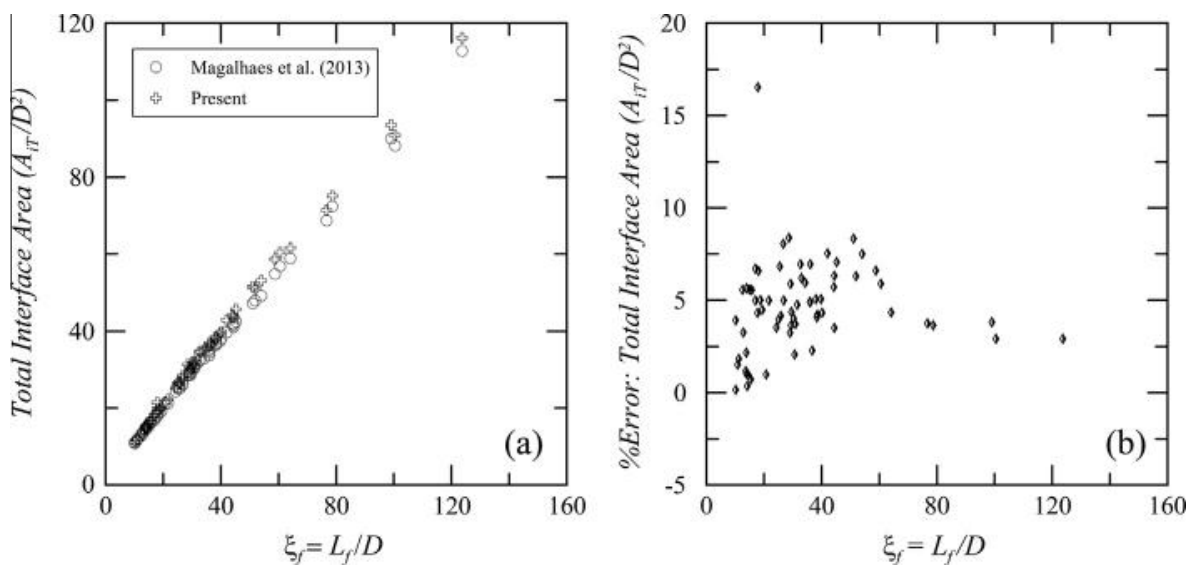

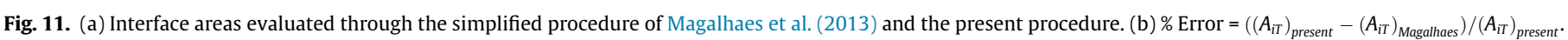


shown. The agreement between experiments and theory in Fig. 10a is very good. In particular, Fig. 10a shows that the length of long bubbles can be correlated with volumes through the power-law expression $L_{f} / D=3.12\left(V^{1 / 3} / D\right)^{2.48}$. Because long bubbles approximate the forms of slender cylinders (limited by the cross section of the pipe), the exponent approaches 3, indicating an almost linear relation between $L_{f}$ and $V$.

For the interfaces, overall predictions are reasonable (Fig. 10b). Fagundes Netto et al. (1999) show that for small Froude numbers $\left(F r_{U}<1\right)$, the interface perturbations have a constant wavelength and a decreasing amplitude. Hence, for very long bubbles, the relative increase in interface area provoked by the perturbations account for a small amount, of the order of 3\%. For the range of Froude number studied in the present work, the difference between experiments and theory for interface area prediction is about $6 \%$.

The total interface area can be reasonably described through the power-law expression $A_{i T} / D^{2}=3.70\left(V^{2 / 3} / D^{2}\right)^{1.16}$, as shown in Fig. 10b.

The best fits in Fig. 10 were found by searching for the maximum coefficient of determination, R-squared. Other statistical parameters were also observed, the residual sum of squares and the residual mean square. In Fig. 10a, the coefficient of determination and the residual mean square were 0.98858 and 0.00411 respectively. In Fig. 10b, these values were 0.98891 and 0.00349 .

\section{Conclusion}

The present work has experimentally identified the complex structure of gas-liquid interfaces in slug flows. The work shows that the mean position of the gas-liquid interface is well characterized by the theory of Fagundes Netto et al. (1999), but that complexities resulting from interface instabilities make the theoretical area predictions about $6 \%$ below the actual values.

The areas and volumes of long bubbles in horizontal slug flows are not normally reported in literature. The present contribution has characterized bubbles with lengths varying from 4 to 130 pipe diameter. The Shadow Sizer technique has been used together with an image treatment procedure implemented in the software Mathematica $^{T M}$.
Two correlations are advanced to parameterize lengths, interface areas and volumes in horizontal slug flows. The non-dimensional expressions have a power-law form and take as reference length the pipe diameter. Other non-dimensional lengths based on the flow dynamics were tried; however, results were not satisfactory.

In the outset, we explained that the interface results of Magalhaes et al. (2013) were obtained through a simplified procedure that basically considered a long bubble to be an axially sectioned cylinder. Fig. 11 compares the present more detailed procedure with the simplified procedure. The percentage error for bubbles as long as $\xi_{f}=50$ can be as high as 8 (Fig. 11b). For longer bubbles, the error appears to be about $3 \%$.

\section{Acknowledgements}

In the course of the research, JBRL benefited from a CNPq Research Fellowship (Grant No 301172/2010-2) and from further financial support through Grants CNPq 477354/2011-4 and FAPERJ E-26/102.212/2013. APSF is grateful to the Brazilian National Research Council (CNPq) for the award of a Research Fellowship (Grant No 303982/2009-8). The work was financially supported by CNPq through Grants No 477293/2011-5 and by the Rio de Janeiro Research Foundation (FAPERJ) through Grant E-26/ $102.937 / 2011$.

\section{References}

Clift, R., Grace, J.R., Weber, M.E., 2005. Bubble, Drops and Particles, Dover, New York, 384p.

Duboc, B., Quiniou, J., Roques-Carmes, C., Tricot, C., Zucker, S., 1989. Evaluating the fractal dimension of profiles. Phys. Rev. A. 39, 1500-1512.

Fagundes Netto, J.R., Fabre, J., Peresson, L., 1999. Shape of long bubbles in horizontal slug flow. Int. J. Multiphase Flow 25, 1129-1160.

Magalhaes, G.R., Goncalves, G.F.N., Loureiro, J.B.R., Silva Freire, A.P., 2013. An experimental investigation of the effects of gas solubility on the properties of horizontal slug flow. Int. J. Multiphase Flow 50, 33-40.

Mandhane, J.M., Gregory, G.A., Aziz, K., 1974. A flow pattern map for gas-liquid flow in horizontal pipes. Int. J. Multiphase Flow 1, 537-553.

Mayor, T.S., Pinto, A.M.F.R., Campos, J.B.L.M., 2007. An image analysis technique for the study of gas-liquid slug flow along vertical pipes. Flow Meas. Instrum. 18, 139-147.

Wallis, G.B., 1969. One Dimensional Two-Phase Flow. McGraw-Hill, New York, 409p. 